\title{
Dynamics of Rotating Multi-Component Turbomachinery Systems
}

Charles Lawrence

Lewis Research Center

Cleveland, Ohio

Prepared for the

34th Structures, Structural Dynamics, Materials Conference sponsored by the American Institute of Aeronautics and Astronautics

La Jolla, California, April 19-21, 1993

(NASA-TM-105997) DYNAMICS OF ROTATING MULTI-COMPONENT TURBOMACHINERY SYSTEMS (NASA) 



\title{
DYNAMICS OF ROTATING MULTICOMPONENT TURBOMACHINERY SYSTEMS
}

\author{
Charles Lawrence \\ National Aeronautics and Space Administration \\ Lewis Research Center \\ Cleveland, Ohio 44135
}

\begin{abstract}
The ultimate objective of turbomachinery vibration analysis is to predict both the overall, as well as component dynamic response. To accomplish this objective requires complete engine structural models, including multi-stages of bladed disk assemblies, flexible rotor shafts and bearings, and engine support structures and casings. In the present approach each component is analyzed as a separate structure and boundary information is exchanged at the inter-component connections. The advantage of this tactic is that even though readily available detailed component models are utilized, accurate and comprehensive system response information may be obtained. Sample problems, which include a fixed base rotating blade and a blade on a flexible rotor, are presented.
\end{abstract}

\section{Intraduction}

A multitude of methods currently are available for the dynamic simulation of turbomachinery blading. Most methods employ simplifying assumptions to ease the analysis process. Few simulation capabilities exist which are capable of treating both the complex geometrical and material configurations of modern day blading as well as the rotational effects associated with turbomachinery. The reason for this limitation is the incapacity of the existing rotational dynamic codes to accommodate the very large number of coordinates required to model the complex geometries, and the inability of the general finite element programs (F.E.), which can handle large complex configurations, to include all of the rotational effects. As a result of this situation, there is limited understanding about the effects of rotation on the elastic vibrations of turbomachinery components.

The ultimate objective of turbomachinery vibration analysis is to predict the overall, as well as individual component, dynamic response. To accomplish this objective, complete engine structural models are required, including multistages of bladed disk assemblies, flexible shafts and bearings, and engine support structures and casings. Clearly, analysis of such systems would require immense modelling capabilities and computational efforts.

Two alternative approaches may be proposed for complete engine vibration analysis. For the first approach, simplifying approximations and reduced order structural models are used to reduce the complexity and computational effort required for the analysis. For example, rotordynamics specialists may consider a system constructed of a flexible shaft with a rigid disk, or a multiple number of rigid disks, each disk having translational and rotational inertias. Disk flexibility effects are assumed negligible.

Bladed-disk specialists, on the other hand, deal with flexible disks, but normally assume rotordynamic effects are negligible. For many real designs these assumptions are valid, however, with new designs, which often are lighter, more flexible, and have more complex geometries and interactions, it is becoming unclear as to which effects are important and therefore must be included for reliable structural response simulations. While the use of simplified approximation approach may lead to reasonable estimations of the actual vibratory response, important phenomena may be overlooked, and detailed response at discrete locations may not be captured.

The second approach entails using complete engine component models, and then applying a "building-block" approach in the analysis. For this approach, each component is analyzed as a separate structure and boundary information is exchanged at the intercomponent connections. The advantage of this tactic is that both comprehensive overall system response is obtained and readily available detailed component models are utilized.

The purpose of the present work is to study blade vibrations in a general framework which includes rotational effects, as well as the blade's interactions with its supporting structures. A finite element basis will be used since this is the most complete and commonly used blade analysis technique currenty available. Rotational effects will be included by supplementing the traditional finite element property matrices with rotational terms. The influence of structural attachments, such as shaft and disk flexibilities, will be included by applying sufficient boundary conditions. Both transient and steady state solution capabilities will be supplied.

In comparison to the large number of studies performed on turbomachinery components, ${ }^{1}$ relatively few undertakings have addressed the problems associated with complete engine vibration analysis. For turbomachinery, bladed disk interactions have been studied frequently. ${ }^{2}$ Cyclic symmetry, mistuning, aeroelastic analysis, and steady state response, among others, are all areas which have been investigated. Another commonly examined system is the coupled shaft- 
disk. In Ref. 3 two radial appendages, used to simulate the disk, were attached to a flexible shaft and critical speeds were determined to vary with appendage flexibility. In Ref. 4 a uniform disk, which is modeled by its one-nodal diameter mode, is attached to a flexible shaft, and resonant speeds are computed. Reference 5 presents a nonrotating multidisk on a shaft finite element model while Ref. 6 evaluates the effect of varying blade, disk, shaft flexibilities on resonant frequencies. Reference 7 examines flexible disks and shaft as well as support structure asymmetry. In Refs. 8 and 9 a transient vibration analysis of a shaft with a flexible disk disturbed by unbalance is studied.

While numerous engine component interaction studies have been made, few address the problem of developing general analytical schemes for complete engine analysis. One of the more extensive vibration codes which has been developed for turbomachinery vibration analysis may be found in Ref. 10 . This modal based code, although limited to constant rotational speed and simplified disks, performs both steady state and transient analysis of engine systems consisting of flexible disks, shafts, bearings, and engine supports. Maybe the most comprehensive development for entire engine vibration analysis is found in Ref. 11. In this reference a complete, and general, scheme is presented for coupling finite element, or modal, models of blades, disks, shafts, and any other relative engine components into a system analysis. The formulation developed in this reference is used in the present study for generating the equations of motion of the blade.

\section{Derivation of Component Equation of Motion}

For practical vibration analysis it is desirable to utilize existing component models, which in many cases are generated from finite elements. As a result, it is beneficial to provide a general component formulation which can accommodate rotational effects within a finite element framework.

The momentum of motion in a body-fixed (commonly used finite element assumption) coordinate system (Fig.1) is written as:

$$
\bar{G}=[M] \dot{\vec{x}}
$$

where $M$ is the body-fixed (rotating) mass matrix, defined in the body-fixed coordinate system, and $\dot{\bar{x}}$ is a vector of absolute velocities. The absolute velocity is the sum of the body-fixed velocities resulting from elastic deformation plus the base velocities resulting from motions at the component boundary where it is connected to the rest of the structure. For continuity, the consistent mass matrix generated from the finite element based formulation is used.

Expanding Eq. (1) by partitioning with respect to finite element nodes:

$$
\bar{G}=\left[\begin{array}{ccccc}
M_{11} & M_{12} & M_{13} & \cdots & M_{1 n} \\
M_{21} & M_{22} & & & \vdots \\
\vdots & & \ddots & & \vdots \\
N_{n 1} & \cdots & \cdots & \cdots & M_{n n}
\end{array}\right]\left\{\begin{array}{c}
\dot{\bar{x}}_{1} \\
\dot{\bar{x}}_{2} \\
\vdots \\
\dot{\bar{x}}_{n}
\end{array}\right\}
$$

$n$ is the total number of nodes in the body. Notice that for a consistent mass formulation there normally is inertial coupling between translations and rotations and between adjacent nodes. While this coupling may be accurately represented by a lumped mass matrix with sufficient nodal refinement for nonrotating systems, it presently is unclear whether the lumped mass is adequate for rotating systems.

Following the strategy of Ref. 11, which is repeated here for clarity, the absolute velocity of node $i$ is:

$$
\dot{\bar{x}}_{\mathrm{i}}=\left\{\begin{array}{c}
\vec{V}+\left[\Omega_{o}\right]\left(\bar{r}+\delta \bar{u}_{x}\right) \\
\bar{W}
\end{array}\right\}_{i}+\left\{\begin{array}{l}
\delta \dot{\bar{u}}_{x} \\
\delta \dot{\bar{u}}_{\theta}
\end{array}\right\}_{i}
$$

where $\delta \vec{u}_{x}$ and $\delta \vec{u}_{\theta}$ are the elastic displacements and rotations with respect to the body-fixed system, $\bar{r}$ is the position of the node from the body-fixed origin, and $\vec{V}$ and $\vec{W}$ are the linear and angular velocities of the body-fixed coordinate system with respect to any inertial system.

The $\Omega_{0}$ term in Eq. (2) is a cross product matrix operator defined as:

$$
\left[\Omega_{0}\right]=\left[\begin{array}{ccc}
0 & -w_{z} & w_{y} \\
w_{z} & 0 & -w_{x} \\
-w_{y} & w_{x} & 0
\end{array}\right]
$$

where $w_{x}, w_{y}$, and $w_{z}$ are rotation rates of the body-fixed coordinate system in body-fixed coordinates. Using the following transformation from inerial to body-fixed, the base rotations are defined as:

$$
\begin{gathered}
\bar{w}=\left\{\begin{array}{l}
w_{Y} \\
w_{Y} \\
w_{3}
\end{array}\right\}=\left[\begin{array}{ccc}
\cos \theta_{Z} & \sin \theta_{Z} & \theta_{X} \sin \theta_{Z}-\theta_{Y} \cos \theta_{Z} \\
-\sin \theta_{Z} & \cos \theta_{Z} & \theta_{X} \cos \theta_{3}+\theta_{Y} \sin \theta_{Z} \\
\theta_{Y} & -\theta_{X} & 1
\end{array}\right] \\
\left\{\begin{array}{c}
\dot{\theta}_{X} \\
\dot{\theta}_{Y} \\
\dot{\theta}_{Z}+\Omega
\end{array}\right\}=[R] \dot{\theta}_{C}
\end{gathered}
$$

where $\theta_{X}, \theta_{Y}$, and $\theta_{Z}$ are rotations of the inertial system, and $\Omega$ is the nominal rotational speed. $R$ is a transformation matrix from inertial to body fixed coordinates. 
The translational and rotational velocity vectors in Eq. (2) are defined as:

$$
\begin{aligned}
& \bar{V}=\left\{\begin{array}{cccc}
R & \left\{\begin{array}{lll}
\dot{X} & \dot{Y} & \dot{Z}
\end{array}\right\}^{T} \\
\bullet & & \\
\bullet & & \\
\bullet & &
\end{array}\right\} \\
& \bar{W}=\left\{\begin{array}{ccc} 
& \bullet & \\
& \bullet & \\
R \quad\left\{\dot{\theta}_{X}\right. & \dot{\theta}_{Y} & \left.\left(\dot{\theta}_{Z}+\Omega\right)\right\}^{T}
\end{array}\right\} \\
& \delta \bar{u}=\left\{\begin{array}{l}
\delta \bar{u}_{x} \\
\delta \bar{u}_{\theta}
\end{array}\right\} \\
& \bar{r}=\left\{\begin{array}{ccc}
\left\{\begin{array}{ccc}
r_{x} & r_{y} & r_{z}
\end{array}\right\}^{T} & \bullet & \\
& \bullet & \\
\bullet &
\end{array}\right\} \\
& \text { and } \Omega_{1}=\left[\begin{array}{cc}
\Omega_{0} & 0 \\
0 & 0
\end{array}\right]
\end{aligned}
$$

Substituting the above terms into Eq. (2), and then into Eq. (1) yields:

$$
\bar{G}=M\left\{\vec{V}+\vec{W}+\Omega_{1}(\vec{r}+\delta \vec{u})+\delta \dot{\bar{u}}\right\}
$$

Differentiating Eq. (6) with respect to time and noting that $M$ is defined in a rotating coordinate system:

$$
\begin{aligned}
\frac{d \bar{G}}{d t}= & M\left\{\dot{\bar{V}}+\dot{\bar{W}}+\dot{\Omega}_{1}(\bar{r}+\delta \vec{u})+\Omega_{1} \delta \dot{\bar{u}}+\delta \ddot{\bar{u}}\right\} \\
& +\Omega_{2} M\left\{\bar{V}+\bar{W}+\Omega_{1}(\bar{r}+\delta \bar{u})+\delta \dot{\bar{u}}\right\}
\end{aligned}
$$

$$
\text { where: } \Omega_{2}=\left[\begin{array}{cccc}
\Omega_{0} & & & \\
& & & -0- \\
& \Omega_{0} & & \\
-0- & \ddots & \\
& & & \Omega_{0}
\end{array}\right]
$$

Completing the equations of motion by including both internal and external forces:

$$
\begin{gathered}
M \delta \ddot{\bar{u}}+C \delta \dot{\bar{u}}+K \delta \bar{u}=M\left\{\dot{\bar{V}}+\dot{\vec{W}}+\dot{\Omega}_{1}\right. \\
\left.(\bar{r}+\delta \bar{u})+\Omega_{1} \delta \dot{\bar{u}}\right\}+\Omega_{2} M\left\{\bar{V}+\bar{W}+\Omega_{1}\right. \\
(\bar{r}+\delta \bar{u})+\delta \dot{\bar{u}}\}+\bar{F}_{E X T}
\end{gathered}
$$

where $C$ and $K$ are damping and stiffness matrices, and $F_{E X T}$ is a vector of externally applied forces.

At this point it is informative to examine Eq. (9) for three special cases.

(1) Fixed Base $(\vec{V}=\vec{W}=\dot{\bar{V}}=\dot{\bar{W}}=0)$. For this case the base of the component is constrained, meaning that the supporting structure is assumed infinitely rigid and therefore does not contribute to the dynamics of the component (e.g., flexible blade on a rotating rigid shaft). For this case Eq. (9) reduces to:

$$
\begin{aligned}
M \delta \ddot{\bar{u}}+ & \left\{C-M \Omega_{1}-\Omega_{2} M\right\} \delta \dot{\bar{u}} \\
+ & \left\{K-M \dot{\Omega}_{1}-\Omega_{2} M \Omega_{1}\right\} \delta \bar{u}=M \dot{\Omega}_{1} \bar{r} \\
& +\Omega_{2} M \Omega_{1} \bar{r}+F_{E X T}
\end{aligned}
$$

(2) Fixed base, constant rotational speed $(\Omega=$ constant, $\dot{\Omega}=0$ ).

$M \delta \ddot{\bar{u}}+\left\{C-M \Omega_{1}-\Omega_{2} M\right\} \delta \dot{\bar{u}}$

$+\left\{K-\Omega_{2} M \Omega_{1}\right\} \delta \bar{u}=\Omega_{2} M \Omega_{1} \vec{r}+F_{E X T}$

(3) Fixed base, constant rotational speed, and lumped Mass (translational only). For this case $M \Omega_{1}=\Omega_{2} M$ and the velocity and displacement terms associated with $M$ reduce to the well known gyroscopic and centrifugal softening terms, respectively.

$$
\begin{gathered}
\mathrm{M} \delta \ddot{\bar{u}}+\{\mathrm{C}-2 \Omega \mathrm{M}\} \delta \dot{\vec{u}}+\left\{\mathrm{K}-\Omega^{2} \mathrm{M}\right\} \delta \bar{u} \\
=\Omega^{2} \mathrm{M} \overline{\mathrm{r}}+\mathrm{F}_{\mathrm{EXT}}
\end{gathered}
$$

\section{Sample Problem One}

The first sample problem is used to assess both the effect of displacement and velocity dependent rotational terms, and 
lumped and consistent mass matrices on the free vibration modal frequencies. A simplified blade model is used so that a physical understanding of the effects of each of the terms may better be obtained. It is particularly useful to study a model where each of the radial, in-plane bending, and out-of-plane bending modes are elastically decoupled.

The blade model consists of a fixed base, uniform beam, having both radial and bending degrees of freedom (Fig. 2). The beam is rotating with a constant rotational speed as shown in the figure. Five beam type finite elements, which contain both elastic and geometric stiffness, are used to model the blade. The model has 4 nodes and 24 degrees of freedom. First, Eq. (9c) (lumped mass) is utilized tocalculate natural frequencies. Then, the effect of consistent mass is studied via Eq. (9b).

Figure 3 shows the effect of geometric stiffness on the modal frequencies as a function of rotational speed (centrifugal softening terms are not included). The geometric stiffness is computed from the centrifugal load acting on the blade due to rotation, then inserting the force into the geometric stiffness matrix. As depicted in the figure, the geometric stiffness causes the bending frequencies to increase with speed, while the radial mode remains constant. Since the beam has identical properties for both in-plane and out-of-plane bending, they are equally effected by geometric stiffening, and thus their frequencies increase the same. The radial mode remains unchanged with increasing speed because there are no geometric stiffness terms associated with the radial degrees of freedoms. (i.e., The frequency of linear spring remains constant regardless of how much it is stretched.) Both the radial and bending mode shapes do not change, at all, with speed.

The results shown in Fig. 4 include geometric stiffening affects, as well as displacement dependent (centrifugal softening) rotational effects. The softening terms, which act in the two in-plane perpendicular directions ( $y-z$, Fig. 2), decrease the modal frequencies for both the radial and in-plane bending modes. The in-plane bending modes, although considerably reduced, continue to increase with speed because of the strength of the geometric stiffening. The radial mode, on the other hand, decreases with speed because it is uninfluenced by geometric stiffness. Ultimately, the radial mode becomes unstable at higher speed.

The results shown in Fig. 5 include geometric stiffness and velocity dependent (gyroscopic) rotational effects. The softening terms are not included. The gyroscopic terms, which act in the same two perpendicular directions as the softening terms, differ from the softening terms in that the gyroscopics are cross coupled. In other words, the gyroscopic force in one perpendicular direction is dependent on the velocity in the other direction, and vice a versa. The effect of the gyroscopic terms is to stiffen both the in-plane bending modes and the radial mode. Because of the coupling between in-plane modes, the radial and bending modes become almost indistinguishable. As with the softening effect only results, the out-of-plane modes are unaffected by the gyroscopics.

Figure 6 depicts the results of including geometric stiffness, centrifugal softening, and gyroscopics. In this case, all of the modes become stiffer with speed, except for the first in-plane bending mode. This is in contrast to the previous results where only the radial mode showed a decrease in frequency as a result of the softening effects. In fact, the radial mode, which approached instability at high speed when softening alone was considered, actually increases in frequency. This increase is a result of the gyroscopic coupling between this mode and the in-plane bending modes. The decrease in stiffness of the first in-plane bending mode also is a result of the in-plane gyroscopic coupling. For the in-plane bending mode, the coupling effect is to decrease the frequency. Another result of the coupling, is the decrease in correlation between the rotating and nonrotating mode shapes. This is particularly evident at higher speeds. For example, at a nondimensional rotational speed of 6 , the radial and second bending in-plane modal correlation is only 30 and 60 percent, respectively. This lack of correlation is indicative of sizable changes in the mode shapes.

All of the results presented thus far have been obtained by using a mixed consistent and lumped mass formulation. While a consistent mass matrix was used for the coefficient of the acceleration vector, a lumped mass matrix was used for the velocity and displacement terms (Eq. 9). For the results in Fig. 7, the lumped mass matrix was replaced by the consistent mass matrix for all coefficient matrices. The results in Fig. 7 are very similar to those produced by using lumped mass (Fig. 6). Altough not distinguishable in the figure, there actually is a 15 percent difference in the first in-plane mode at high speed (nondimensional speed $=6$ ). Also, beyond a speed of 2 , there are no modes that correlate with the nonrotating radial mode. In other words, the purely radial mode has vanished.

Figures 8 to 10 show how each of the in-plane modes are affected by the inclusion of the various rotational terms. The out-of-plane modes are not included because they are affected only by geometric stiffness. Figure 8 depicts the radial mode's independence from geometric stiffness, softening from displacement dependent terms, and stiffness due to gyroscopics. Figure 9 shows the first in-plane bending modes decrease in frequency due to combined softening and gyroscopics, and increase in frequency from all other effects. Figure 10 presents the second in-plane bending mode's increase in stiffness due to all combinations of rotational effects.

The present example has demonstrated the interactions between the in-plane radial and bending modes through velocity dependent cross coupling. In particular, the stiffening and softening of the radial and bending modes, respectively, has been shown. A question which arises from these results is whether the radial mode always serves to soften the bending mode, and conversely, whether the bending mode always helps to stiffen the radial mode. In an attempt to setule this issue, the radial and bending modes were inspected for various 
radial to bending mode stiffness ratios. The radial to bending mode stiffness ratio was varied by increasing the blade radial stiffness.

The stiffness ratio's influence on the in-plane radial and first bending modes are shown in Figs. 11 and 12. For the lowest ratio $(r=1)$, where the nonrotating frequency of the radial mode equals that of the bending, the radial mode becomes unstable when the rotational speed reaches the radial mode's frequency. This result signifies a lack of interaction between the radial and bending modes because the identical result is obtained for a single degree of freedom, rotating, linear spring-mass system. Results at $r=1$ are unavailable for the bending mode since the system is radially unstable. At intermediate stiffness ratios ( $r=5$ and 10$)$, the radial and bending modes are coupled, and the stiffening of the radial mode along with the softening of the bending mode, previously seen, are evident. At high stiffness ratios $(r=100)$, the radial mode is unaffected by rotation, and in contrast to lower ratios, no apparent softening effects are transferred from the radial to bending mode. The implication of these results is that bending behavior is unmodified by the radial mode when the radial mode is very stiff, and significantly modified when the radial mode is in the range of the bending mode stiffness.

\section{Sample Problem Two}

The previous sample problem provided insight into the effects of the various rotational terms. Nevertheless, the necessity of including the terms is best determined through actual problems. To accomplish this objective an actual rotating propeller blade, term propfan blade, is analyzed.

The propfan blade, in contrast to conventional propellers, is thin and flexible, of low aspect ratio and high sweep (Fig. 13). As a result of this complex geometry, bending, torsional, and radial modes all are highly coupled. Finite element analysis is required for accurate structural analysis. ${ }^{13}$

The Finite Element program MSC/NASTRAN was used to model the propfan. Use of this program is advantageous because of its capability to model the complex geometry of the propfan as well as analyze the large displacement (geometric nonlinear) response of the flexible blade due to rotation. A limitation which NASTRAN does have is its inability to automatically include centrifugal softening and gyroscopic effects. These additional terms were incorporated manually into a NASTRAN combined geometric/normal modes solution sequence through a special NASTRAN programming language (Ref. 13) and the utilization of Eq. (9c).

Figures 14 to 16 depict the variation of the blade natural frequencies as a function of rotational speed. In Fig. 14 the geometric stiffening effect is shown to increase all six of the blade's fundamental modes. The relationship is nonlinear since the stiffening forces are related to the rotational speed squared. The results in Fig. 15 are generated by including both the velocity (gyroscopic) and displacement (softening) terms. The combined effect of including these terms appears to be relatively small, except at high rotational speeds where there is considerable difference from the results presented in Fig.14. Figure 16 compares the effect of including the softening terms along with the geometric stiffness versus including the geometric terms alone. As displayed in this figure, the softening effect is significant, demonstrating the need to include this term for reliable blade analyses. The gyroscopic terms also were examined but their effect, for this blade, was negligible.

\section{Component Interactions}

In the examples presented thus far single component systems with fixed boundary conditions were studied. To determine the effect of multicomponent interactions, a simple, two degrees of freedom, nonrotating system was used (Fig. 17). Specifically, this system was used to assess the possible range of interactions between a bladed assemblage and a shaft. This system also is identical to a two degrees of freedom vibration isolator. The study of this system is important because it is imperative to recognize the boundary between where isolated component models are reliable, and where coupled system models are required. For this simplified representation of a bladed-assemblage on a shaft, the blade mass is taken as the sum of all blades in the assembly, and the disk mass is added to the shaft.

Figure 18 shows blade and shaft normalized frequencies for varying ratios of blade and shaft frequencies and mass. As demonstrated in the figure, the extent of the coupling effect is dependent on both the blade to shaft mass and frequency ratios. The mass ratio effect is expected since light blades would have little influence over shaft response, and conversely heavy shafts would act similarly to fixed blade supports. Consequently, for small mass ratios decoupled component analyses are suitable.

The effect of blade to shaft frequency ratio is less clear because the coupled system frequencies may increase or decrease depending on whether the ratio is less than or greater than 1.0. For very soft blades $(r=0.01)$ neither the blade or shaft frequencies are effected, regardless of mass ratio. For ratios between 0.01 and 1.0 the blade frequencies decrease with coupling while the shaft frequencies increase. For ratios above 1.0 the opposite effect is seen where the blade frequencies increase and the shaft frequencies decrease. At a ratio of 1.0 , where the blade and shaft frequencies are identical, there is a switching of modes where the blade mode goes from the lower to higher mode, and the shaft mode goes from higher to lower.

The application of these results to real problems may be implemented by considering actual physical masses or by utilizing effective modal masses. By utilizing modal masses, a more accurate blade to shaft mass ratio will be constructed. For example the bending mode of the shaft may be coupled most closely to the blade radial mode in which case the shaft 
bending mode modal mass would be compared to the blade radial mode modal mass. Similarly, for a system where the shaft was relatively stiff, and the blade was coupled to a flexible disk, the modal masses from the blade and disk would be used in the ratio.

\section{Sample.Problem Three}

In this sample problem, the previously presented flexible blade model is coupled to a flexible shaft. The coupled model (Fig. 19) represents an extreme case because a large unbalance is exerted onto the shaft by the single blade. The model consists of 9 beam elements and 11 six-degree-of-freedom nodes. The blade and shaft have separate, but coincident, nodes at the boundary where they are connected. The ratio of blade to shaft modal mass for the first bending mode is 0.15 and the frequency ratio is 1.0 . The entire system is supported on bearings and is spun about the shaft's longitudinal axis.

The previously developed equations of motion (Eq. 9) are used for the dynamic modeling of the blade and shaft. Information then is exchanged at the blade-shaft boundary to couple the blade-shaft dynamics (Fig. 20). As shown in the figure, the blade has influence by exerting boundary forces onto the shaft, and the shaft effects the blade through a combination of boundary displacements, velocities, and accelerations.

Three sets of transient vibration data were generated. The first set (I) included all rotational effects for both the shaft and blade. The second set (II) included centrifugal blade softening and shaft gyroscopics. Shaft centrifugal softening and blade gyroscopics were not included. As previously mentioned, this is a common assumption made by blade and shaft analysts. The third data set (III) included blade and shaft centrifugal forces only. Gyroscopic and softening were not included for the blade or shaft. An externally applied step load was applied todof 4 on the shaft with a direction in the plane of rotation and perpendicular to the blade. A transient dynamic analysis was performed and displacements were computed for each of the three cases.

Figure 21 shows transient displacements for four locations $(4,19,20$, and 21, Fig. 19) on the system for each of the three cases (I to III). The rotation rate was equal to one-fourth of the frequency associated with the blade and shaft first bending mode. DOF 19, which is the blade tip lateral displacement perpendicular to the rotational plane, is unaffected by rotation up to the time when the step load is applied $(T=15 \mathrm{sec})$. Subsequent to this time, the resulting shaft deflection produces out of plane coupling and displacements in the 19 direction. As depicted in the figure, there is minimal difference between case I and II, and significant differences for case III. Figures 21(b) to (d) show similar results for two other blade DOF's and the shaft DOF where the step load is applied.
The results shown in Fig. 22 were generated by repeating the previous transient analyses but at a higher rotational speed. For these results, the rotational speed was increased to onehalf of the first bending mode frequencies. The results in this figure are similar to the previous figure. The main difference is that the absolute displacements are larger because of the larger rotational speed. In general, for all the DOF's, rotational speeds, and cases run, there are insignificant differences between cases I and II. This indicates that the omission of blade gyroscopics and shaft centrifugal softening is a good approximation.

\section{Summary of Results}

1. A practical method for rotating coupled system transient analysis was presented in this paper. This method is advantageous because it is based on FE methodologies and allows for independent modelling and analysis of components.

2. A uniform, rotating, beam analysis was used to determine that, without gyroscopics, centrifugal softening has a destabilizing effect on the radial modealone. When gyroscopics are included, all in-plane modes are coupled. As a result, instabilities, or even stiffening effects, may occur in any of the modes. Out-of-plane modes are affected by neither softening or gyroscopics.

3. Except at very high rotational speeds, the differences in natural frequencies between the consistent and lumped mass matrix is insignificant.

4. From analysis conducted on an actual propeller blade model, it was determined that centrifugal softening effects are significant, and that gyroscopics may be neglected.

5. From a simple, two DOF, nonrotating model used to represent a blade-shaft system, it was found that significant dynamic interactions occur when the blade and shaft frequency ratio, as well as the mass ratios, are of the same order.

6. From a fully coupled, rotating, blade-shaft analysis the importance of shaft gyroscopics, blade softening, and the exchange of loadings at the blade-shaft boundaries, was demonstrated.

\section{References}

1. Rao, J.S., "Turbomachine Blade Vibration," The Shock and Vibration Digest, Vol. 19, No. 5, May 1987, pp. 3-10.

2. Omprakash, V., and Ramamurti, V.,"Analysis Of Bladed Disks-A Review," The Shock and Vibration Digest, Vol. 20, No. 11, Nov. 1988, pp. 14-21.

3. Wilgen, FJ., and Schlack, A.L., 1957, "Effects of Disk Flexibility on Shaft Whirl Stability," AIAA Joumal, 
Vol. 15, No. 10, Oct., 1977, pp. 1531-1533. (Also, ASME Paper 78-WAVDE-4, 1978).

4. Dopkin, J.A., and Shoup, T.E., "Rotor Resonant Speed Reduction Caused by Flexibility of Disks," Journal of Engineering for Industry, Vol. 96, No. 4, Nov. 1974, pp. 1328-1333.

5. Shahab, A.A.S., and Thomas, J., "Coupling Effects of Disc Flexibility on the Dynamic Behavior of Multi Disc-Shaft Systems," Lournal of Sound and Vibration, Vol. 114, No. 3, May 1987, pp. 435-452.

6. Palladine,J.A., and Rosettes, J.N., "Finite Element Analysis of the Dynamics of Flexible Disk Rotor Systems," ASME Paper 82-GT-240, 1982.

7. Klompas, N., "Theory of Rotor Dynamics with Coupling of Disk and Blade Flexibility and Support Structure Asymmetry," ASME Paper 74-GT-159, 1974.

8. Sakata, M., Aiba, T., and Ohnabe, H., "Transient Vibration of High Speed Lightweight Rotor Due to Sudden Imbalance," ASME Paper 82-GT-231, 1982.

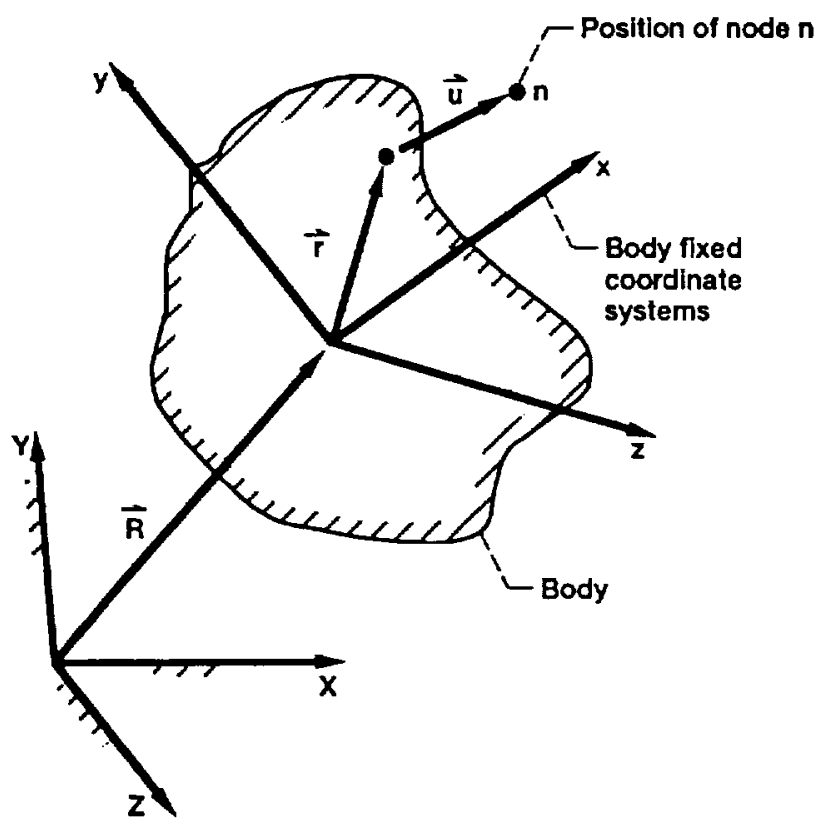

Figure 1.-Coordinate system deflnitions.
9. Klompas, N. "Unbalance Response Analysis of a Complete Turbomachine," Joumal of Enrineering forPower, Vol. 105, No. 1, Jan., 1983, pp. 184-191.

10. Galardo, V.C., and Black, G., "Blade Loss Transient Dynamics Analysis," Vol. 1, Task II: "TETRA 2 Theoretical Development," NASA CR-179632, 1986.

11. Davis, R.R., "Practical Nonlinear Simulation of Rotating Machinery Dynamics With Application to Turbine Blade Rubbing," PhD Dissertation, University Of Califomia, Davis, CA, 1989.

12. Mehmed, O., and Kaza, K.R.V., "Experimental Classical Flutter Results of a Composite Advanced Turboprop Model," NASA TM-88792, 1986

13. MSC/NASTRAN Programmer's Manual, Vol. III. Macneal-Schwendler Corp., Los Angeles, CA, 1983.

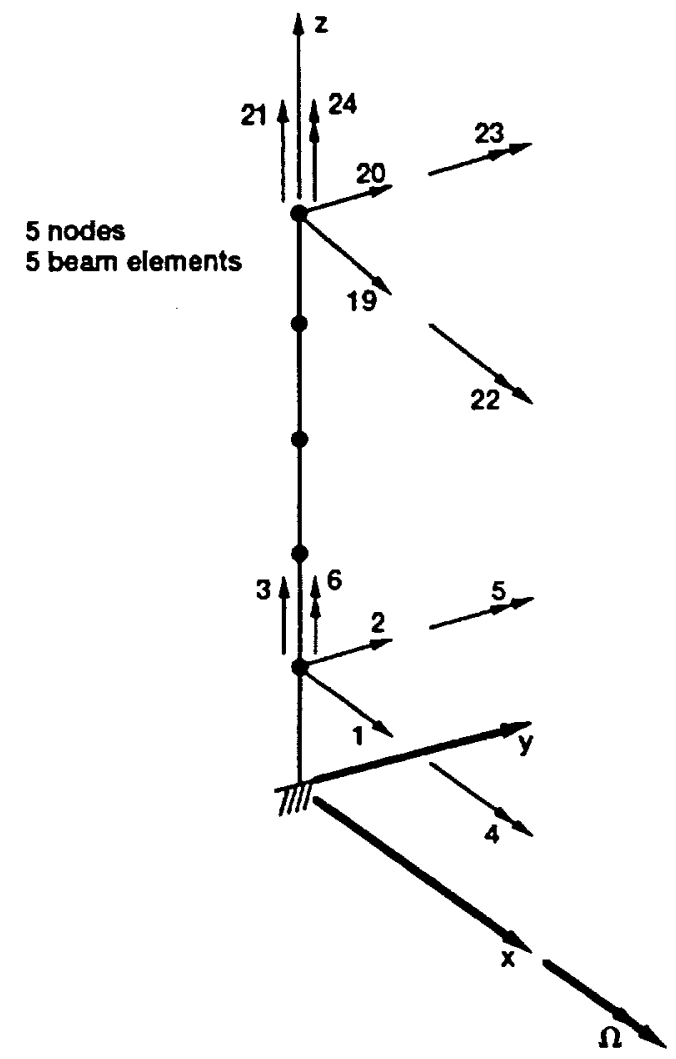

Figure 2.-Finite element blade model. 


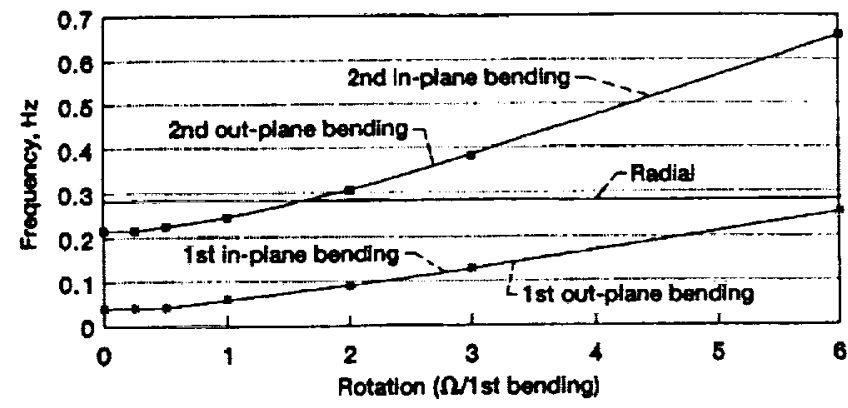

Figure 3.-Natural frequency versus rotational speed-geometric stiffness only. (Radial frequency/bending trequency $=8$ ).

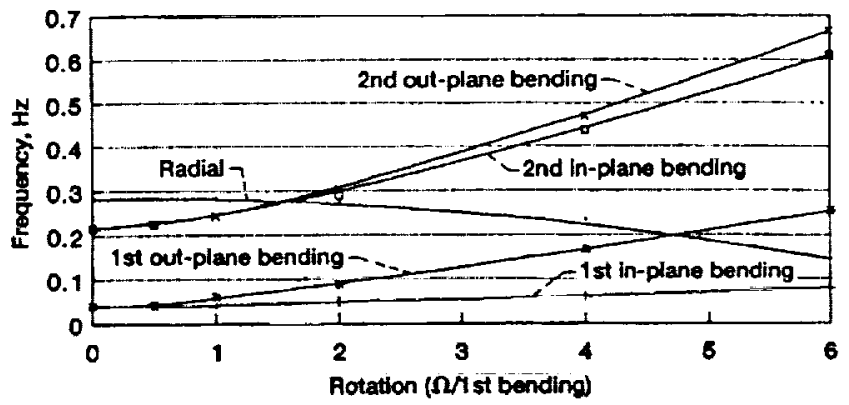

Figure 4.-Natural frequency versus rotational speed-geometric and softening included. (Radial frequency/bending frequency $=8$ ).

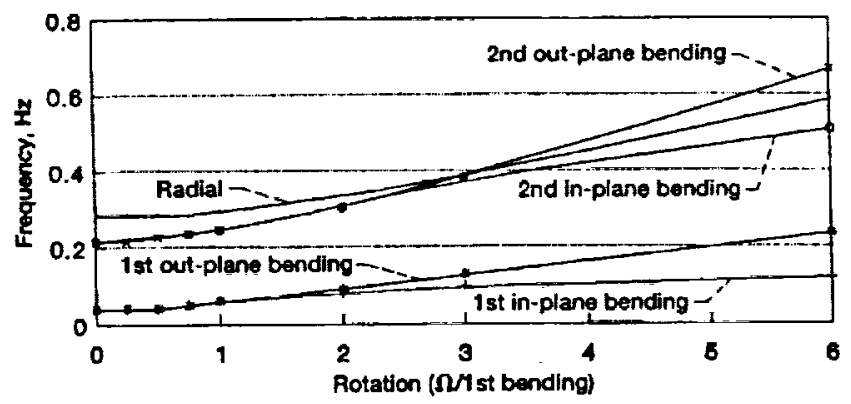

Flgure 5.-Natural frequency versus rotational speed-geometric stiffness and gyroscopics included. (Riadial frequency/ bending frequency $\mathbf{~} 8$ ).

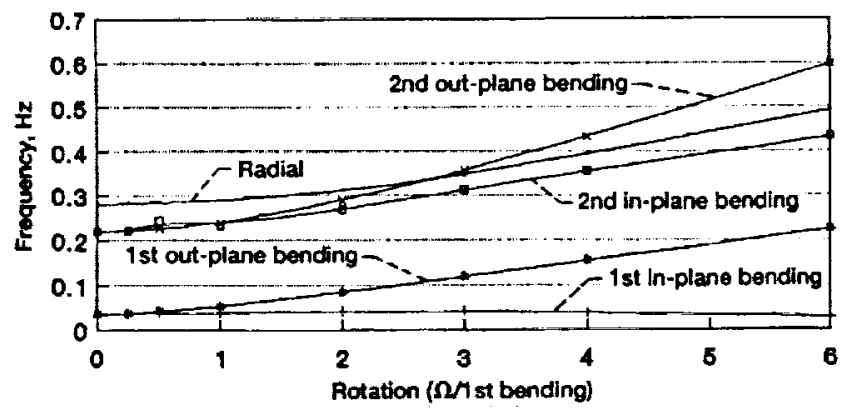

Figure 6.-Natural frequency versus rotational speed-geometric stiffness, sottening, and gyroscopics included. (Radial trequency/bending frequency $=8$ ).

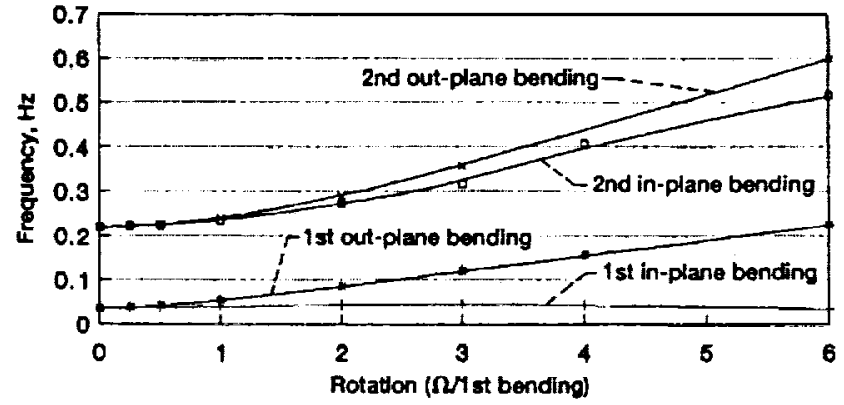

Figure 7.- Natural frequency versus rotational speed-consistent mass. Nelocity and displacement included).

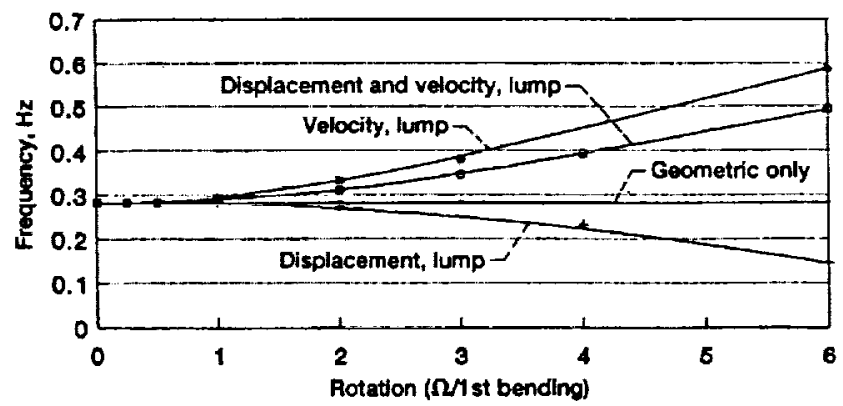

Figure 8.-Natural frequency versus rotational speed-radial mode.

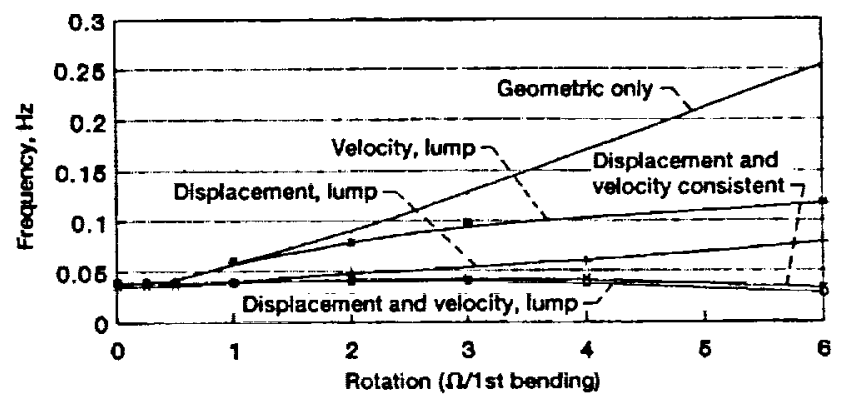

Figure 9.-Natural frequency versus rotational speed- $f_{1}$ inplane.

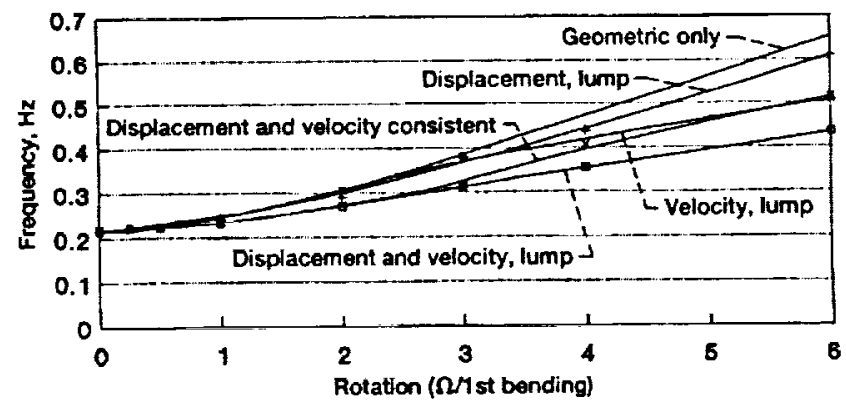

Figure 10.-Natural frequency versus rotational speed- $\hbar_{2}$ inplane. 


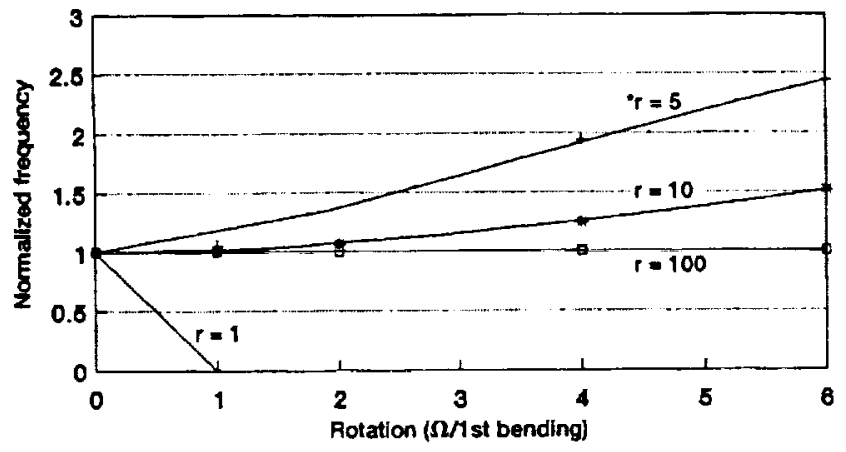

- $r$ = radial mode frequency/1st bending mode frequency.

Figure 11. - Natural frequency versus rotational speed-radial mode. (Lumped mass, softening and gyroscopics included).

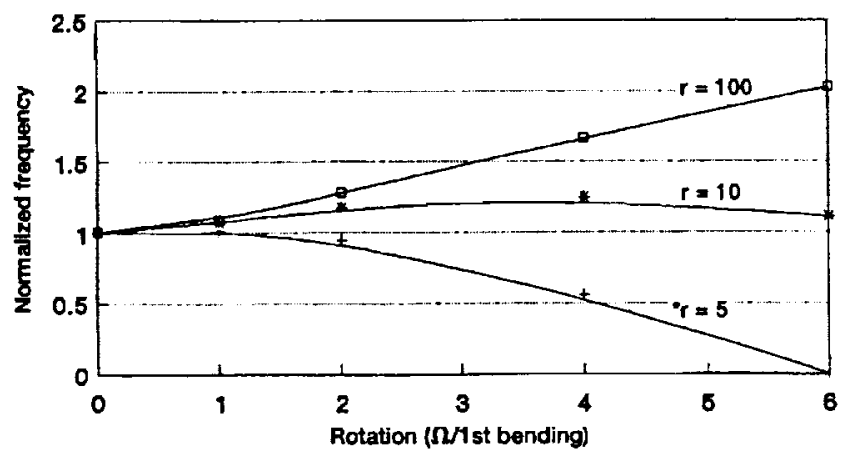

" $r$ = radial mode frequency/1st bending mode frequency.

Figure 12.-Natural frequency versus rotational speed-1st inplane bending. (Lumped mass, displacement and velocity terms included).

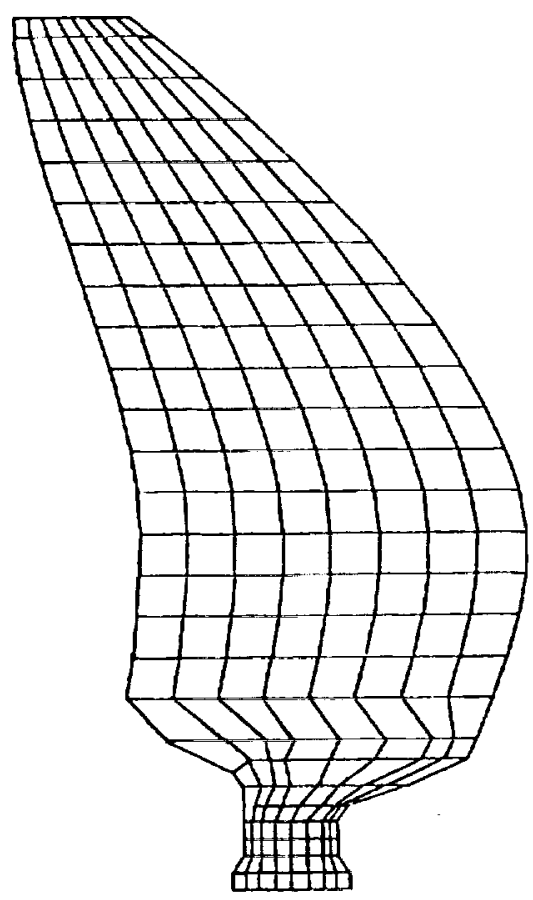

Figure 13.-Propfan blade finite element model.

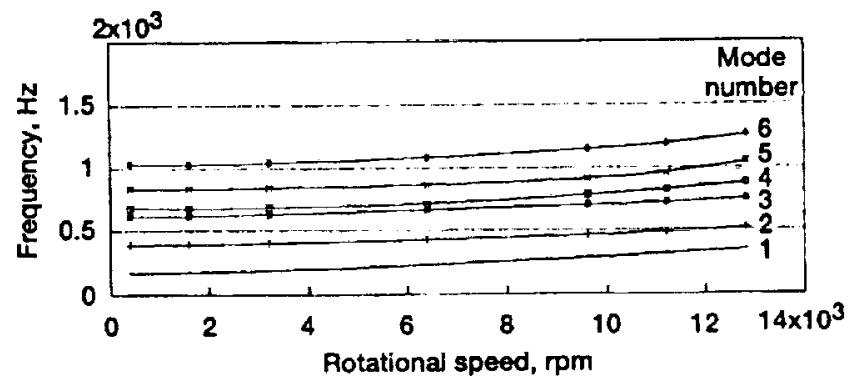

Figure 14.-Propfan blade natural frequency versus rotational speed. (Geometric stiffness only).

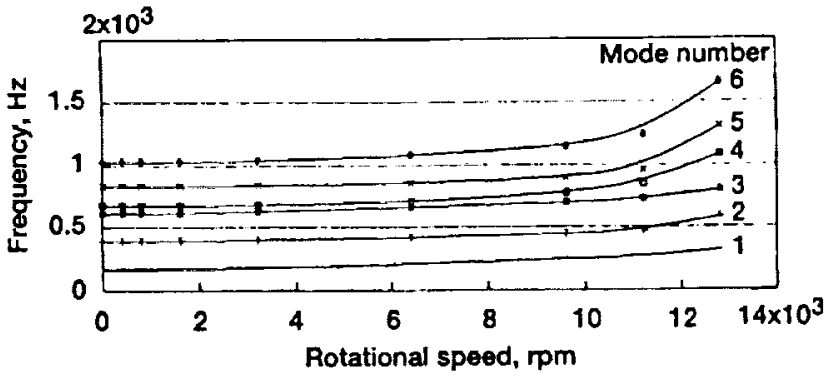

Figure 15.-Propfan blade natural frequency versus rotational speed. (Geometric stiffness, centrifugal softening and gyroscopics included).

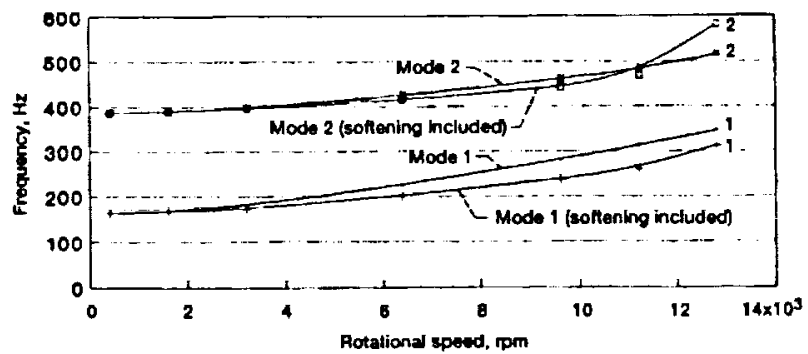

Figure 16. - Propfan blade natural frequency versus rotational speed. Mode 1 and 2. 


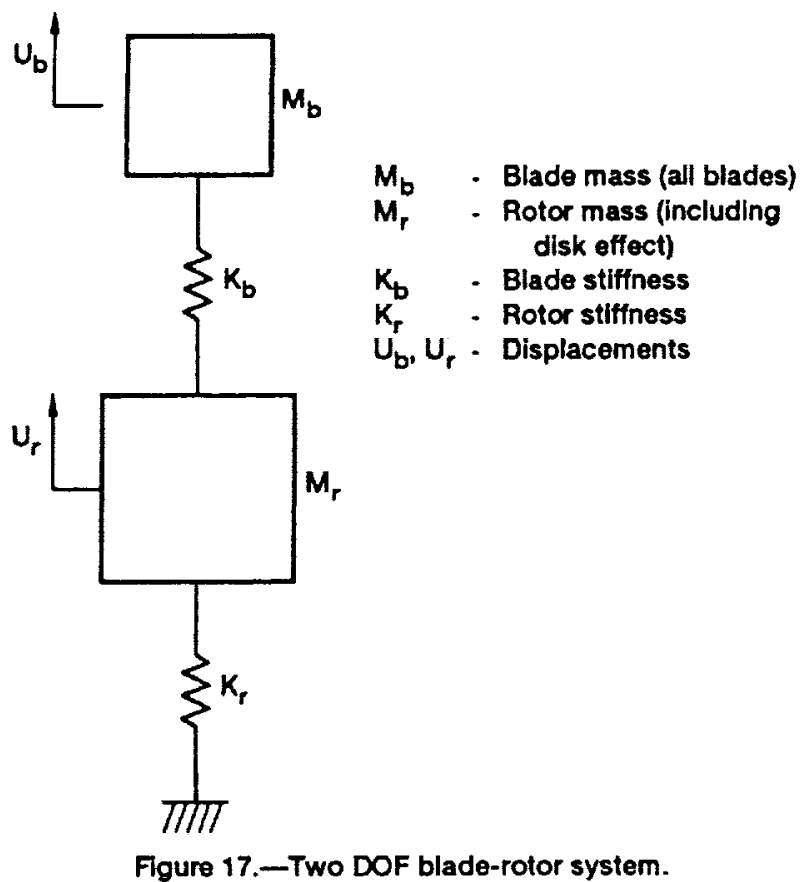

Figure 17. - Two DOF blade-rotor system.
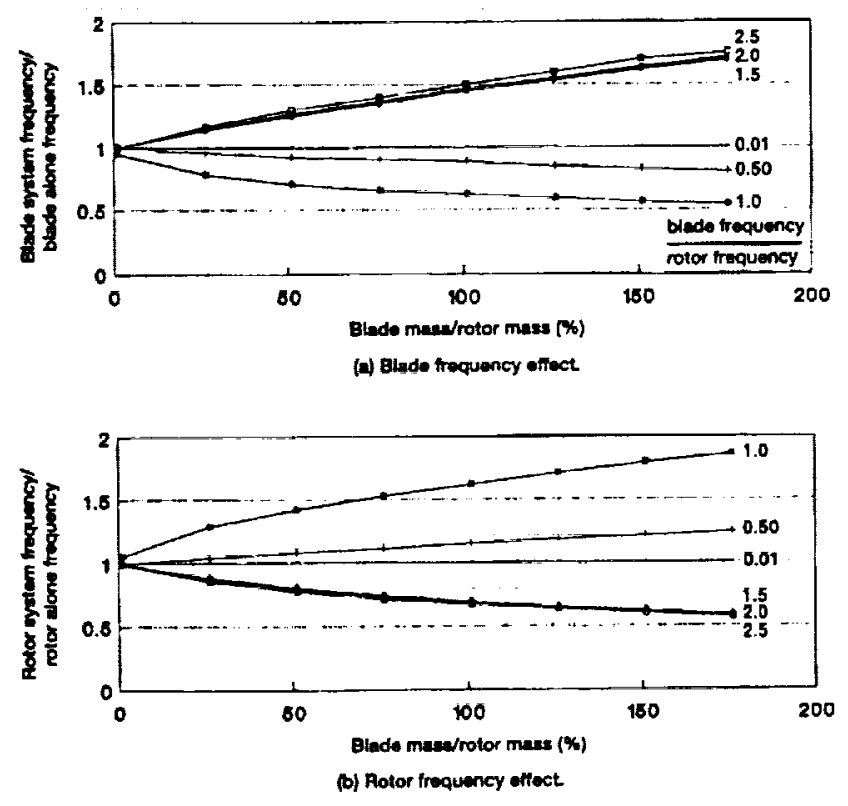

Figure 18.-Blade-rotor interaction. $(\Omega=0)$.

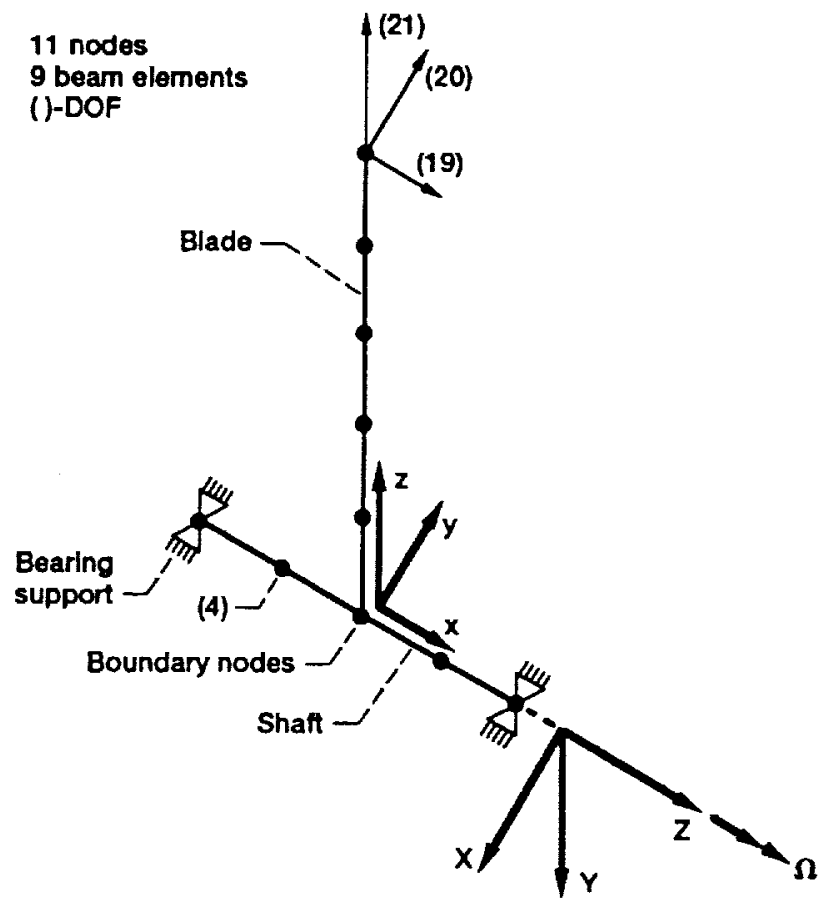

Figure 19.-Coupled system finlte element model.

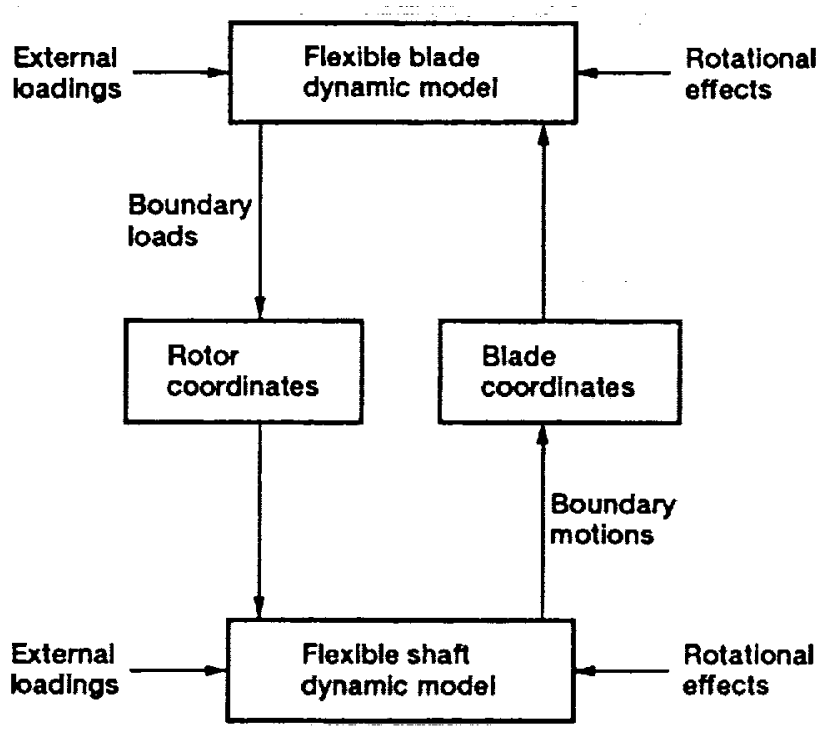

Figure 20.-Blade-shaft dynamic coupling scheme. 


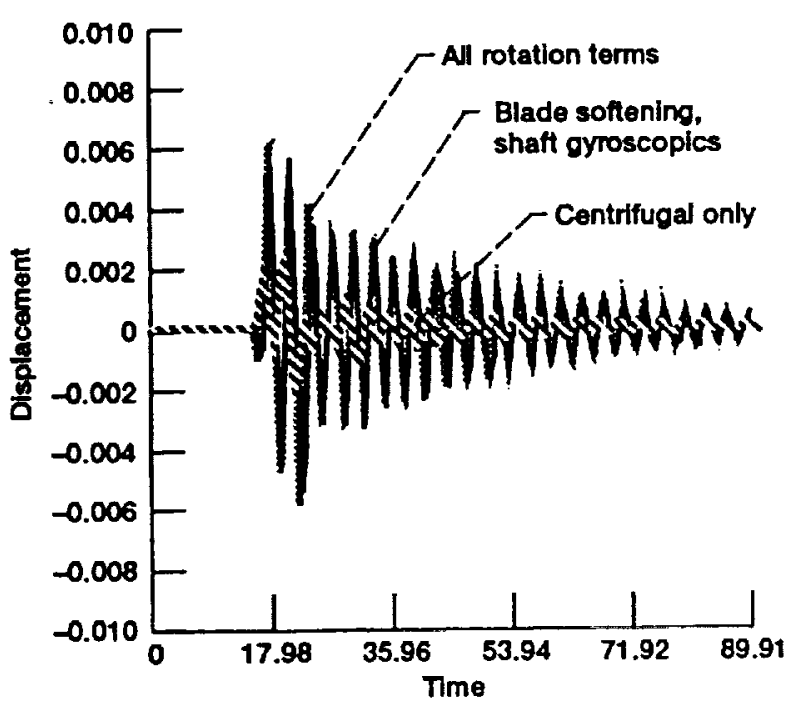

(a) DOF 19.

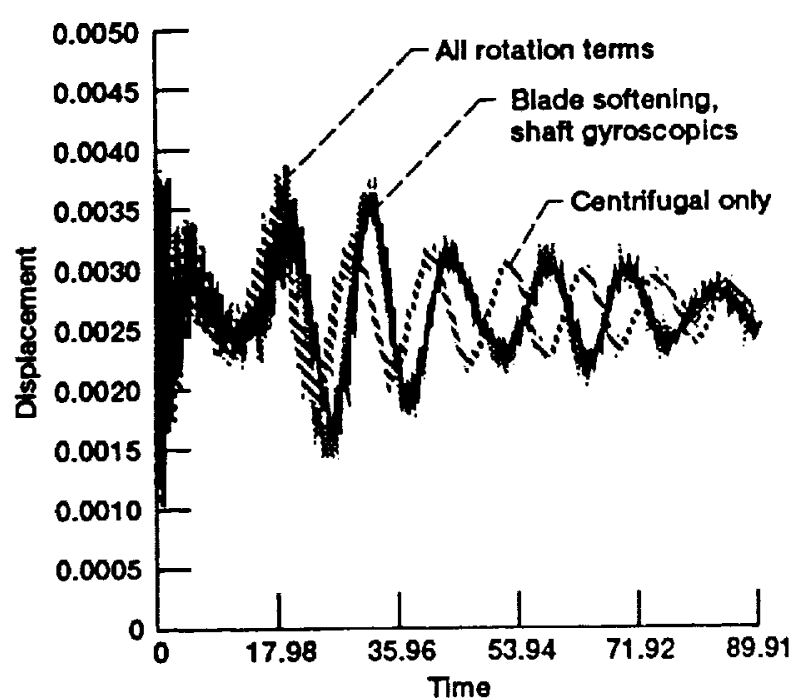

(c) DOF 21.

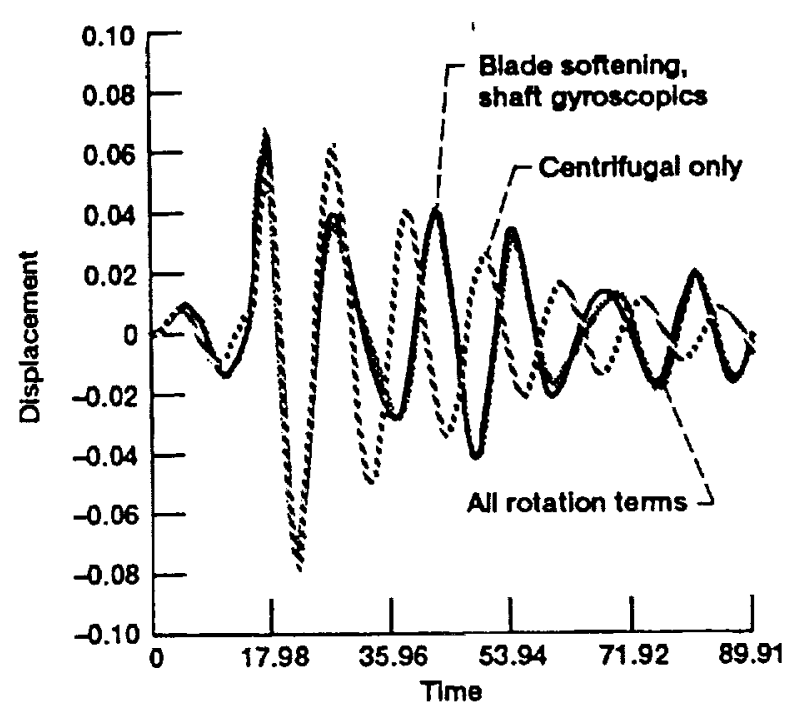

(b) DOF 20.

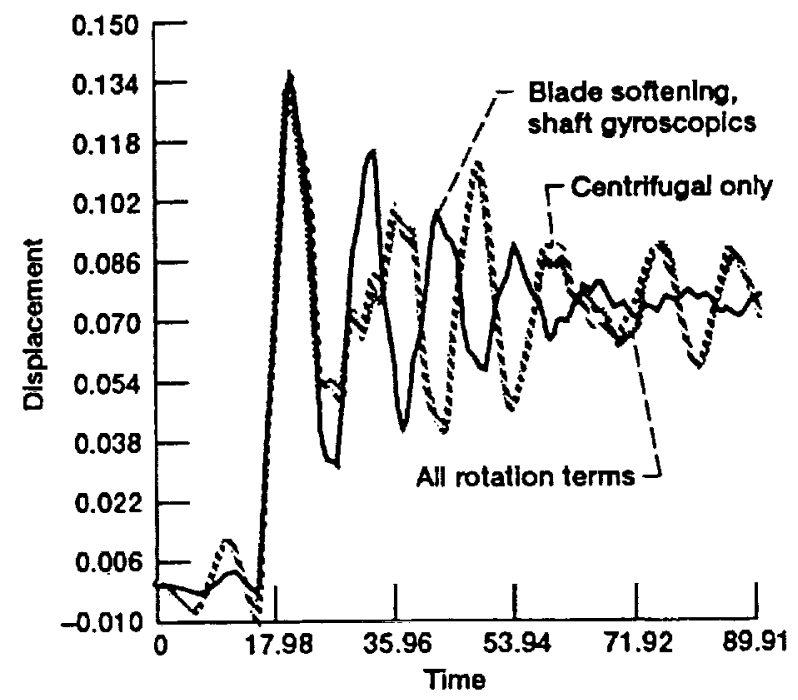

(d) DOF 4.

Figure 21. Transient response due to rotation and step load on shaft ( $\Omega=0.25 f_{1}$ ) 


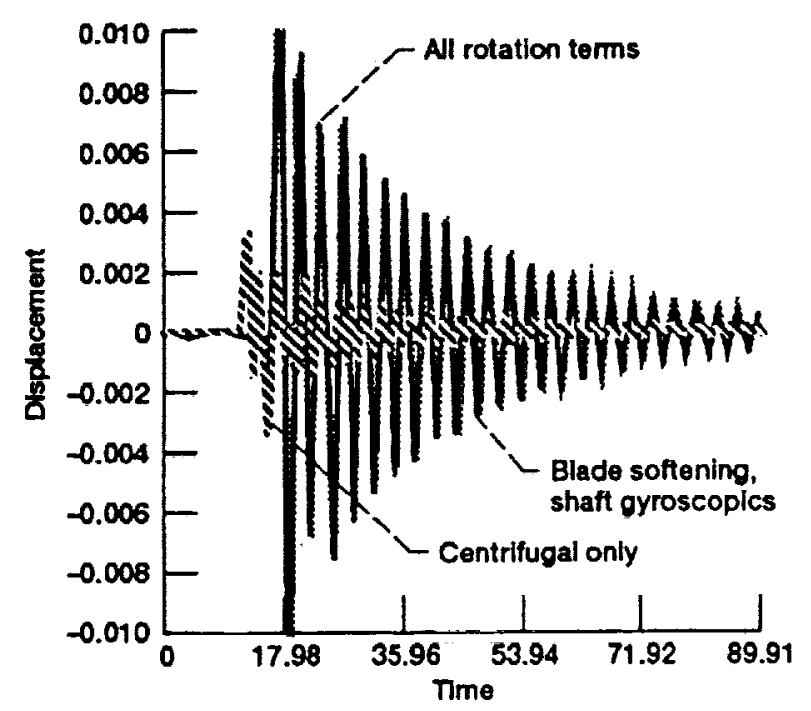

(a) DOF 19.

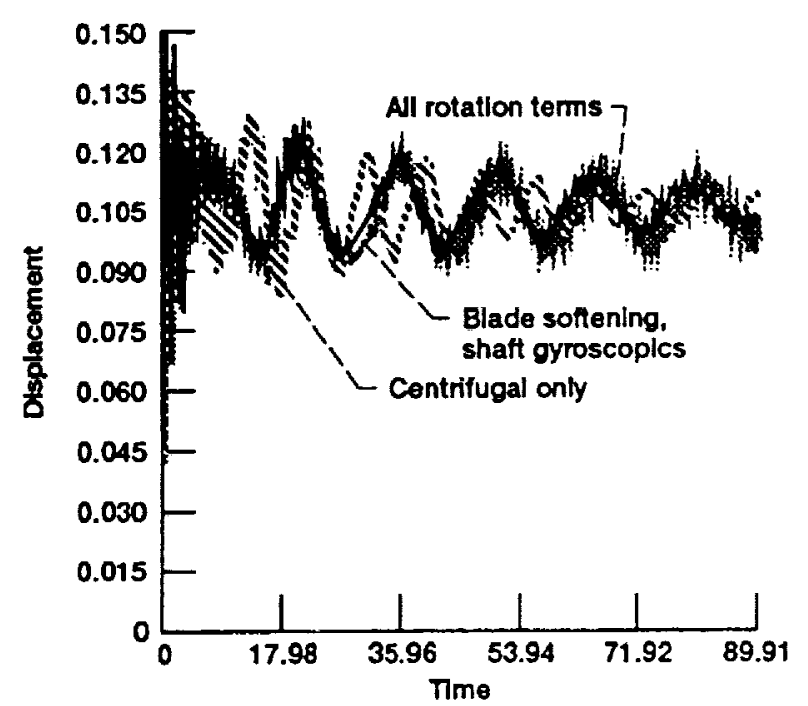

(c) DOF 21.

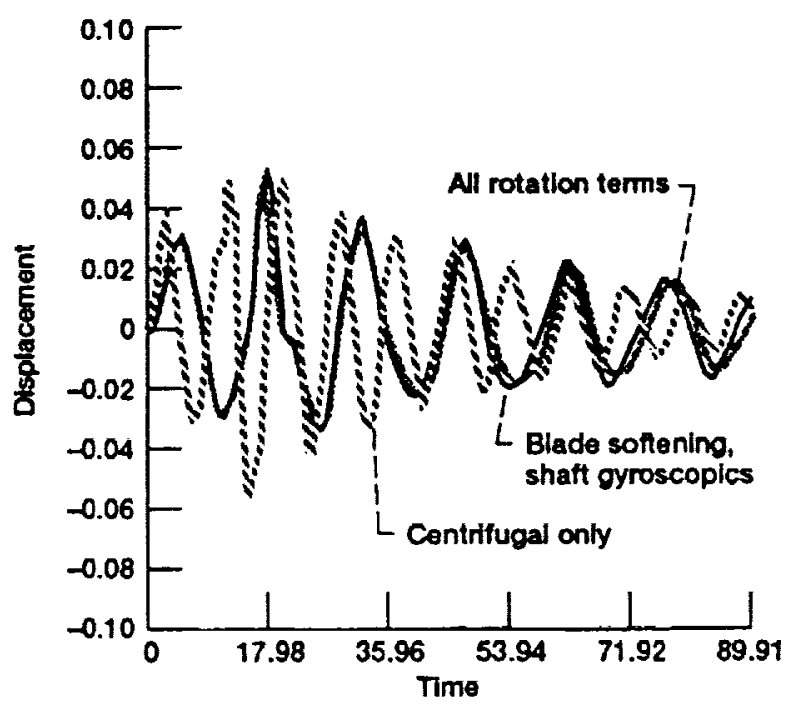

(b) DOF 20.

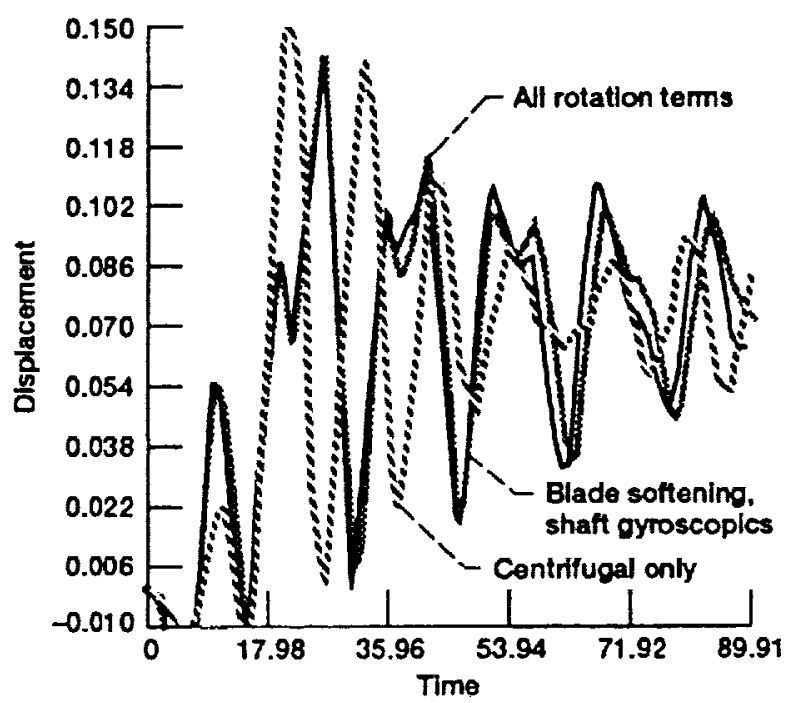

(d) DOF 4.

Figure 22. -Transient response due to rotation and step load on shaft ( $\Omega=0.50 t_{1}$ ) 


\begin{tabular}{|c|c|c|c|}
\hline \multicolumn{3}{|c|}{ REPORT DOCUMENTATION PAGE } & $\begin{array}{l}\text { Form Approved } \\
\text { OMB No. 0704-0188 }\end{array}$ \\
\hline \multicolumn{4}{|c|}{ 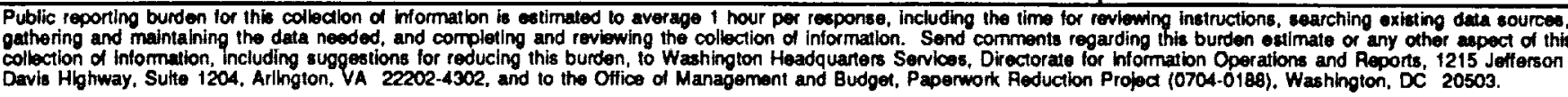 } \\
\hline 1. AGENCY USE ONLY (Leave blank) & $\begin{array}{l}\text { 2. REPORT DATE } \\
\text { January } 1993\end{array}$ & 3. $\mathbf{R}$ & $\begin{array}{l}\text { DD DATES COVERED } \\
\text { echnical Memorandum }\end{array}$ \\
\hline \multicolumn{3}{|c|}{$\begin{array}{l}\text { 4. TILE AND SUBTाLE } \\
\text { Dynamics of Rotating Multi-Component Turbomachinery Systems }\end{array}$} & 5. FUNDING NUMBERS \\
\hline \multicolumn{2}{|l|}{ 6. AUTHOA(S) } & & WU-505-63-53 \\
\hline \multicolumn{3}{|c|}{$\begin{array}{l}\text { National Aeronautics and Space Administration } \\
\text { Lewis Research Center } \\
\text { Cleveland, Ohio } 44135-3191\end{array}$} & $\begin{array}{l}\text { 8. PERFORMNG ORGANHZATION } \\
\text { REPORT MUMBER } \\
\text { E-7537 }\end{array}$ \\
\hline \multicolumn{3}{|c|}{$\begin{array}{l}\text { National Aeronautics and Space Administration } \\
\text { Washington, D.C. 20546-0001 }\end{array}$} & $\begin{array}{l}\text { 10. SPONSORING/MONTORING } \\
\text { AGENCY REPORT NUMBER } \\
\text { NASA TM-105997 }\end{array}$ \\
\hline \multicolumn{4}{|c|}{$\begin{array}{l}\text { 11. SUPPLEMENTARY NOTES } \\
\text { Prepared for the 34th Structures, Structural Dynamics, Materials Conference sponsored by the American Institute of } \\
\text { Aeronautics and Astronautics, La Jolla, Califomia, April 19-21, 1993. Responsible person, Charles Lawrence, } \\
\text { (216) 433-6048 }\end{array}$} \\
\hline \multicolumn{3}{|c|}{$\begin{array}{l}\text { 120. DISTRIBUTIONAVAILABILTY STATEMENT } \\
\text { Unclassified - Unlimited } \\
\text { Subject Category } 39\end{array}$} & 12b. DISTRIBUTION CODE \\
\hline
\end{tabular}

\section{ABSTRACT (Maximum 200 words)}

The ultimate objective of turbomachinery vibration analysis is to predict both the overall, as well as component dynamic response. To accomplish this objective requires complete engine structural models, including multistages of bladed disk assemblies, flexible rotor shafts and bearings, and engine support structures and casings. In the present approach each component is analyzed as a separate structure and boundary information is exchanged at the inter-component connections. The advantage of this tactic is that even though readily available detailed component models are utilized, accurate and comprehensive system response information may be obtained. Sample problems, which include a fixed base rotating blade and a blade on a flexible rotor, are presented.

\begin{tabular}{|c|c|c|}
\hline \multicolumn{3}{|l|}{ 14. SUBJECT TERMS } \\
\hline $\begin{array}{l}\text { 17. SECUATY CLASSIFICATION } \\
\text { OF REPORT } \\
\text { Unclassified }\end{array}$ & $\begin{array}{l}\text { 18. SECURTY CLASSIFICATION } \\
\text { OF THIS PAGE } \\
\text { Unclassified }\end{array}$ & $\begin{array}{l}\text { 19. SECURTTY CLASSIFICATION } \\
\text { OF ABSTRACT } \\
\text { Unclassified }\end{array}$ \\
\hline
\end{tabular}

